\title{
A new approach to the identification of distortion modes of thin-walled structures based on plate/shell theory
}

\author{
Lei Zhang ${ }^{1}$, Weidong $\mathrm{Zhu}^{2}$, Aimin $\mathrm{Ji}^{1}$ and Liping Peng ${ }^{1}$ \\ ${ }^{1}$ College of Mechanical and Electrical Engineering, Hohai University, Changzhou 213022, China \\ ${ }^{2}$ Department of Mechanical Engineering, University of Maryland, Baltimore County, MD 21250, USA
}

\begin{abstract}
In this paper, a new approach to identify cross-section deformation modes is presented and utilized in the establishment of a high-order beam model for dynamic analyses of thin-walled structures. Towards this end, a systematic procedure to extract cross-section in-plane vibration shapes for a thin-walled cross-section is developed based on elastic plate/shell theory. Then the distortion shapes are separated from vibration shapes by removing the components of classic modes involved with the minimum value problem of 2-norm. Sequentially, curve fitting method is utilized to approximate the distortion shape functions along the cross-section midline. It should be noticed that these distortion modes are arranged in hierarchy consistent with the order that they are identified and the number of distortions to be identified depends on the required model precision. Based on this, Hamilton's principle is applied to formulate the dynamic governing equations of the beam by constructing its displacement field with the linear superposition of the cross-section mode shapes including distortions. Numerical examples are also presented to validate the new approach and to demonstrate its efficiency in the reproduction of three-dimensional behaviours of thin-walled structures in dynamic analyses.
\end{abstract}

\section{Introduction}

Thin-walled structures are widely used in civil and mechanical engineering. For the simplicity and the ease of applying optimization algorithms coupled with computational efficiency, one-dimensional theories are more preferred than two- and three-dimensional models. However, the development of an efficient onedimensional theory faces a fundamental challenge: a general and easy-to-implement procedure to identify the cross-section modes [1]. Ideally, this procedure should lead to a hierarchical set of modes, in the sense that only the first few modes should be sufficient to describe the deformed configuration with an "acceptable precision" [2]. In other words, the core issue is to reduce the threedimensional continuum elasticity formulation to a beam model which is as accurate as possible to include the most characteristic structural behaviors with the least amount of cross-section modes.

To support this, several trends of enhancing beam theories have been developed in the last decades: asymptotical methods, such as variational asymptotical sectional analyses by $\mathrm{Yu}$ and Hodges [3], expansion of the beam displacement field through Taylor series [4] and refinement of the classic thin-walled theories by Vlassov (in particular the theory of Kim et al. [5]). In engineering, other formulations are deduced considering specific structural behaviors, such as the multi-cell distortion (Gonçalves et al. [6]) and warping due to shear- lag effects (Chen et al. [7]). Moreover, some studies are focused on physically meaningful section modes [8].

Currently, the generalized beam theory is considered as one of the most complete theories, which has been developed to account for both warping and distortion with shear deformation [9] and transverse extension included, being also applied to multi-cell closed crosssections [10] and composite materials [11]. A comparable theory is the method of Generalized Eigenvectors [12], whose modes are obtained through a cross-section discretization using two-dimensional elements. It is also remarkable that Vieira et al. [13, 14] have proposed a high order one-dimensional model being an alternative to those theories. It is credible that these theories are powerful enough to deal with almost any arbitrary thinwalled cross-sections with an acceptable precision. However, the only problem is the solution of nonlinear or generalized eigenvalues, which is quite involved in uncoupling sectional deformation modes [15]. Actually, a practicable method being not so full-featured but with high precision is more suitable in some cases.

A one-dimensional high-order beam model for thinwalled structures is presented in this paper with a novel approach to identify distortion modes. This model is thought to consider a projection of the displacement field on the beam cross-section through a set of linear independent basis functions. For the mathematically impaired, basis functions can be considered as the mode shape functions of cross-section modes. To obtain a 
hierarchical set of cross-section modes, the approach is first carried out by building a shell/plate model of the thin-walled structure for modal analyses with its crosssection vibration shapes extracted. These vibration shapes are deemed as the linear superposition of cross-section mode shapes. In this point, a set of cross-section modes can be identified from the cross-section vibration shapes by uncoupling the foregone cross-section modes. Then the residual nodal displacements are employed to define new distortion modes with the curve fitting method. In this process, the basis functions are approximated over the cross-section, guaranteeing the compatibility of the displacement field along the cross-section midline, and new distortion modes are identified.

\section{One-dimensional formulations}

\subsection{Displacement fields}

Consider a prismatic thin-walled member, such as depicted in Figure 1. The displacement of a point on the midline of the cross-section are defined in terms of the axial $u$, tangential $v$ and normal $w$ components at given $\mathrm{z}$ and the components are prescribed positive along the axial of local coordinate system $(n, s, z)$ adopted in each wall, respectively. Also shown is the global coordinate system $(x, y, z)$ with its origin located in the centroid of the cross-section of the beam end.

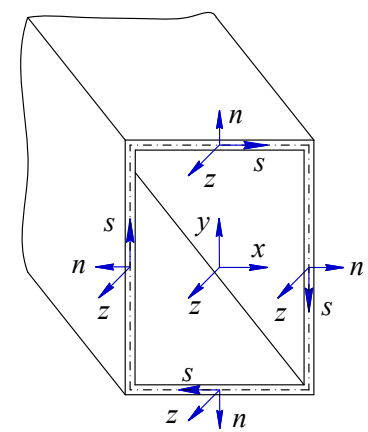

Fig. 1. Global $(x, y, z)$ and local $(s, n, z)$ coordinate systems of a thin-walled structure.

Employing Kirchhoff's hypothesis, the displacement of an arbitrary point $\mathbf{U}(n, s, z)$ is expressed as

$$
\mathbf{U}=\left[U_{z}, U_{s}, U_{n}\right]^{\mathrm{T}}=\left[u-n \frac{\partial w}{\partial z}, v-n \frac{\partial w}{\partial s}, w\right]^{\mathrm{T}}
$$

where the components are approximated independently through a set of linear independent basis functions defined along the coordinate $s$ as

$$
\begin{gathered}
\mathbf{u}=[u(s, z), v(s, z), w(s, z)]^{\mathrm{T}}=\boldsymbol{\psi} \mathbf{x} \\
\boldsymbol{\psi}=\left[\begin{array}{c}
\varphi_{1}(s), \varphi_{2}(s), \cdots, \varphi_{r}(s) \\
\psi_{1}(s), \psi_{2}(s), \cdots, \psi_{r}(s) \\
\phi_{1}(s), \phi_{2}(s), \cdots, \phi_{r}(s)
\end{array}\right]^{\mathrm{T}}, \mathbf{x}=\left[\chi_{1}(z), \chi_{2}(z), \cdots, \chi_{r}(z)\right]^{\mathrm{T}}
\end{gathered}
$$

and the generalized displacements $\chi_{i}(z)$ are to represent the axial variation of $N_{1}$ out-of-plane and $N_{2}$ in-plane amplitude functions $\left(r=N_{1}+N_{2}\right)$, with basis functions $\varphi_{i}(s)$, $\psi_{i}(s)$ and $\phi_{i}(s)$ to describe the cross-section deformation of axial, tangential and normal directions, separately.

Then the three-dimensional displacement $\mathbf{U}$ can be expressed with a transformation matrix $\mathbf{H}$ as

$$
\mathbf{U}=\mathbf{H u}=\left[\begin{array}{ccc}
1 & 0 & -n \frac{\partial}{\partial z} \\
0 & 1 & -n \frac{\partial}{\partial s} \\
0 & 0 & 1
\end{array}\right] \mathbf{u} .
$$

The strain and stress field are written as

$$
\begin{gathered}
\boldsymbol{\varepsilon}=\left\{\begin{array}{l}
\varepsilon_{z z}(n, s, z) \\
\varepsilon_{s s}(n, s, z) \\
\gamma_{s z}(n, s, z)
\end{array}\right\}=\mathbf{C U}, \boldsymbol{\sigma}=\left\{\begin{array}{l}
\sigma_{z z}(n, s, z) \\
\sigma_{s s}(n, s, z) \\
\tau_{s z}(n, s, z)
\end{array}\right\}=\mathbf{E} \boldsymbol{\varepsilon} \\
\text { where } \mathbf{C}=\left[\begin{array}{ccc}
\frac{\partial}{\partial z} & 0 & 0 \\
0 & \frac{\partial}{\partial s} & 0 \\
\frac{\partial}{\partial s} & \frac{\partial}{\partial z} & 0
\end{array}\right], \quad \mathbf{E}=\left[\begin{array}{ccc}
\frac{E}{1-v^{2}} & \frac{E v}{1-v^{2}} & 0 \\
\frac{E v}{1-v^{2}} & \frac{E}{1-v^{2}} & 0 \\
0 & 0 & \frac{E}{2(1+v)}
\end{array}\right], E
\end{gathered}
$$

and $v$ are the material Young's modulus and Poisson's ratio, respectively.

\subsection{Beam governing equations}

The beam energy components are essential for the application of Hamilton Principle, including the strain energy, potential energy and kinetic energy. By definition, they are separately given by

$$
\begin{gathered}
U=\frac{1}{2} \iiint_{V} \boldsymbol{\varepsilon}^{\mathrm{T}} \boldsymbol{\sigma} \mathrm{d} V=\frac{1}{2} \iint_{L} \mathbf{x}_{A}^{\mathrm{T}} \boldsymbol{\psi}^{\mathrm{T}} \mathbf{H}^{\mathrm{T}} \mathbf{c}^{\mathrm{T}} \mathbf{E} \mathbf{c H} \boldsymbol{\psi} \mathbf{x} \mathrm{d} A \mathrm{~d} z \\
U_{\mathrm{P}}=-\iint_{L} \mathbf{U}^{\mathrm{T}} \mathbf{p} \mathrm{d} A \mathrm{~d} z=-\int_{L} \mathbf{x}^{\mathrm{T}} \boldsymbol{\psi}^{\mathrm{T}} \mathbf{H}^{\mathrm{T}} \mathbf{p} \mathrm{d} z \\
T=\frac{1}{2} \iiint_{V} \eta \frac{\partial \mathbf{U}^{\mathrm{T}}}{\partial t} \frac{\partial \mathbf{U}}{\partial t} \mathrm{~d} V=\frac{1}{2} \iint_{L} \int_{A} \frac{\partial \mathbf{x}^{\mathrm{T}}}{\partial t} \boldsymbol{\psi}^{\mathrm{T}} \mathbf{H}^{\mathrm{T}} \eta \mathbf{H} \boldsymbol{\psi} \frac{\partial \mathbf{x}}{\partial t} \mathrm{~d} A \mathrm{~d} z
\end{gathered}
$$

where $V, A$ and $L$ are beam volume, section area and length, separately; $\eta$ is material density; $\mathbf{p}$ denotes a loading vector assumed to be $\mathbf{p}=[p, q, r]^{\mathrm{T}}$ with $p, q, r$ as external loads in axial, tangential and normal directions.

The formulation of the dynamic governing equation involves the application of Hamilton Principle, reading

$$
\delta H=\delta \int_{t_{1}}^{t_{2}} L_{\mathrm{a}} \mathrm{d} t=0,\left.\delta \mathbf{x}\right|_{t=t_{1}}=\left.\delta \mathbf{x}\right|_{t=t_{2}}=0
$$

where $H$ denotes the Hamiltonian, $L_{\mathrm{a}}=T-U-U_{\mathrm{p}}$ is the Lagrangian, and $t_{1}$ and $t_{2}$ are boundary values of time.

Substituting Equations (5), (6) and (7) into (8) yields

$$
\begin{gathered}
\iint_{L} \delta \mathbf{x}_{A}^{\mathrm{T}} \boldsymbol{\psi}^{\mathrm{T}} \mathbf{H}^{\mathrm{T}} \eta \mathbf{H} \boldsymbol{\psi} \frac{\partial^{2} \mathbf{x}}{\partial t^{2}} \mathrm{~d} A \mathrm{~d} z+\int_{L} \int_{A} \delta \mathbf{x}^{\mathrm{T}} \boldsymbol{\psi}^{\mathrm{T}} \mathbf{H}^{\mathrm{T}} \mathbf{c}^{\mathrm{T}} \mathbf{E} \mathbf{c H} \boldsymbol{\psi} \mathbf{x} \mathrm{d} A \mathrm{~d} z \\
-\int_{L} \int_{A} \delta \mathbf{x}^{\mathrm{T}} \boldsymbol{\psi}^{\mathrm{T}} \mathbf{H}^{\mathrm{T}} \mathbf{p} \mathrm{d} A \mathrm{~d} z-\int_{A}\left[\delta \mathbf{x}^{\mathrm{T}} \boldsymbol{\psi}^{\mathrm{T}} \mathbf{H}^{\mathrm{T}} \overline{\boldsymbol{\sigma}}\right]_{z=z_{1}}^{z=z_{2}} \mathrm{~d} A \mathrm{~d} z=0
\end{gathered}
$$

as the dynamic governing equation of thin-walled beams.

\section{Calculation of distortion modes}

\subsection{Shell-like deformations}

The calculation of distortion is based on shell/plate theory. Here a thin-walled beam is discretized into shell/plate elements and then solved for modal analyses. Normally, the member is fixed at one end and the other free. 
Through modal analysis, the first few vibration shapes are derived with displacements at the free end extracted for further study. Here the number of vibration modes studied is determined by the number of distortion needed. And more distortions are needed to improve the precision of the model. In most situations, 4 to 8 modes are essential and may be enough for thin-walled structures with simple cross-sections. Since only distortions are concerned, in-plane components are separated out of the vibration shapes (Figure 2 shows the first 4 modes).

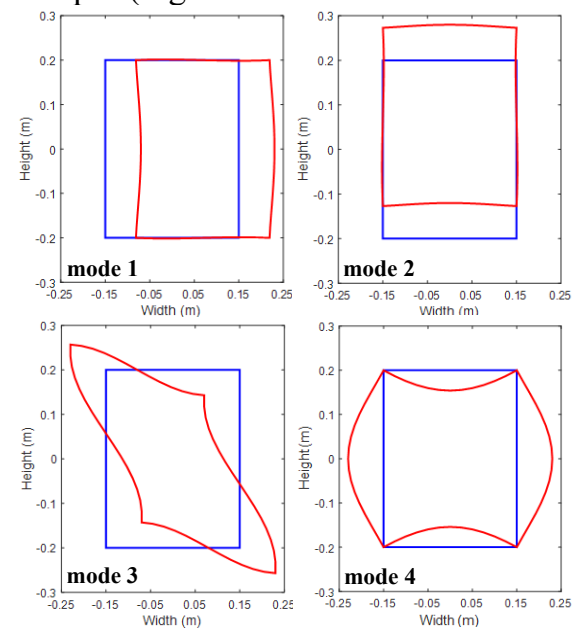

Fig. 2. The sectional in-plane vibration shapes of the first 4 modes of the cantilevered thin-walled structure.

Obviously, those deformed profiles are superposed by cross-section rigid translations and deformation modes. Therefore, the next procedure is pivotal to uncouple the deformation modes by removing rigid translation components, which are usually named as classic modes.

\subsection{Identification of distortion modes}

The identification of distortion is mandatory from the view of enhancing a competitive beam model. This enables the physical perception of the structural phenomenon in a more clear way.

The implementation of this idea requests removing the classic modes (three in-plane modes, namely rotation about $\mathrm{z}$ axis, translations along $\mathrm{x}$ and $\mathrm{y}$ axes, see Figure 3) from the cross-section deformation shapes, with new deformation modes (distortion) left. Then the application of curve fitting is involved to define the shape functions.
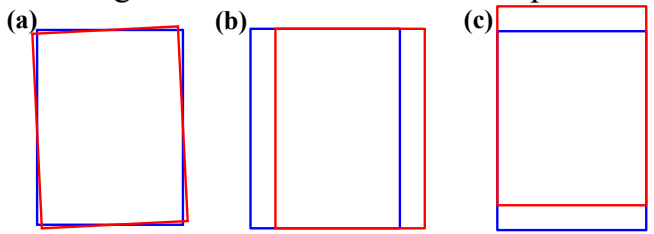

Fig. 3. Classic in-plane mode shapes: (a) rotation about longitudinal $z$ axis; (b) and (c) flexions along $x$ and $y$ axes.

To remove the classic modes from one cross-section vibration shape, one may consider a solution of the form

$$
\left\|\boldsymbol{\Theta}-\kappa_{i} \boldsymbol{\theta}_{i}\right\|_{2}=\min _{\lambda_{i} \in R}\left\|\boldsymbol{\Theta}-\lambda_{i} \boldsymbol{\theta}_{i}\right\|_{2}
$$

where $\Theta$ indicates the vector constructed by the nodal displacement of the cross-section in-plane vibration shape (Figure 2), while $\boldsymbol{\theta}_{i}$ represents that of a classic mode shape; $\lambda_{i}$ is a coefficient spanning the vector space $\boldsymbol{\Phi}=$ span $\left\{\boldsymbol{\theta}_{i}(s)\right\}$ and $\kappa_{i}$ corresponds to the solution which shows the participation of classic modes. For the first several modes, the cross-section deformation shape usually contains one classic and one distortion mode. In this point, the residual vector $\boldsymbol{\Theta}-\kappa_{i} \boldsymbol{\theta}_{i}$ can be used to define a new distortion mode (Figure 4), expressed as

$$
\chi_{j}(s)=\operatorname{polyfit}\left(\boldsymbol{\Theta}-\kappa_{i} \boldsymbol{\theta}_{i}\right)
$$

where $\chi_{j}(s)$ constructs the vector $\mathbf{x}$ in Equation 2.

It should be noted that the procedure to remove the classic modes is not exclusive. Strictly speaking, distortions refer in particular to in-plane tortuosity and twisting of a cross-section, just opposite to the rigid displacements of the whole cross-section (classic modes). Therefore, one may directly get off the rigid displacement components from shell-like deformations with manual visual method. For example, plainly the cross-section flexion along $x$ axis (Figure 3b) participates a lot in the deformed profile shown in mode 1 of Figure 2. One may easily determine the corresponding $\kappa_{i}$ (in Equation 10) according to the displacements of the section corners. Then the residual nodal displacements of the shell/plate model is used for curve fitting to define a new distortion demonstrated as Figure 4a (cubic polynomial fitting). In this way, the participation $\kappa_{i}$ of one classic mode is solved individually from each section deformed profile.

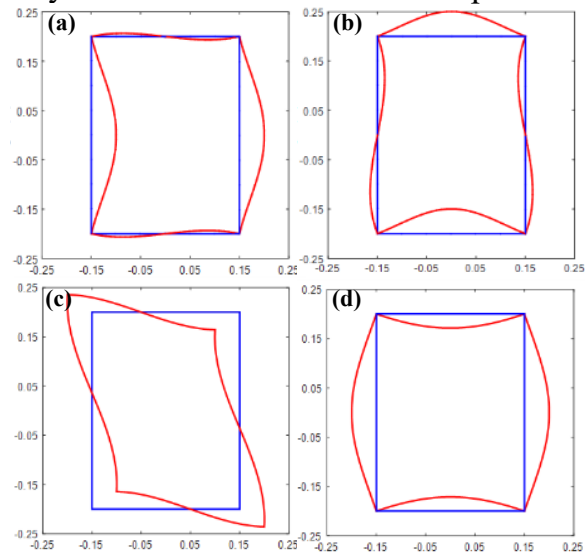

Fig. 4. Distortions of the thin-walled structure: (a) distortion 1; (b) distortion 2; (c) distortion 3 and (d) distortion 4.

\section{Illustrative examples}

Numerical studies are carried out on a cantilevered thinwalled beam with rectangular hollow cross-section (Figure 5) to validate the new approach.

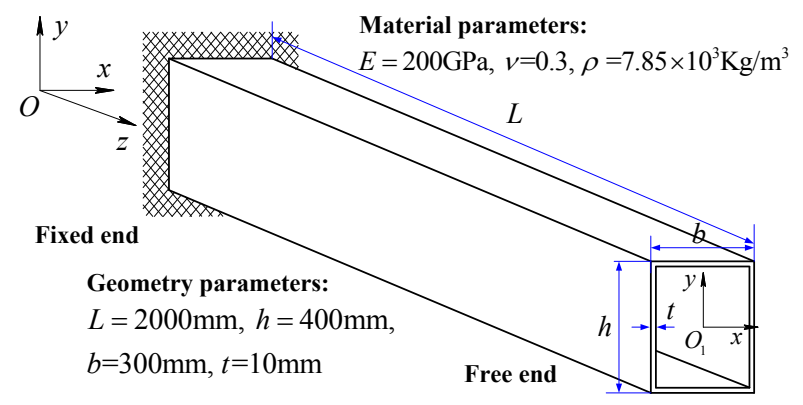

Fig. 5. A cantilevered thin-walled structure with geometry and material parameters as shown. 
The finite element method is involved with linear Lagrange function approximating displacements within an element. Table 1 presents the information about the first 10 modes, consisting of the values of the natural frequencies $f_{i}$, the relative differences $\delta_{i}$ and the distortion mode participations. The results of present model are obtained with 80 equally distributed elements. In ANSYS analyses, the beam is discretized into 4480 Shell 181 4node elements, distributed as 80 elements along the length, 16 along the web height and 12 along the flange width. The modal participations are calculated by

$$
\mu_{i}=\frac{X_{i \max }\left(\phi_{i \max }+\psi_{i \max }\right)}{u_{\text {max }}} \times 100 \%
$$

where $u_{\max }$ is the maximum displacement of a generic point on the free end, while the relative differences are derived based on the assumption that the results derived from ANSYS shell theory are accurate enough.

Table 1. Free vibration results about frequencies concerning the first 10 vibration modes.

\begin{tabular}{cccccccc}
\hline Mode & Present & Ansys & \multicolumn{4}{c}{ Distortion mode } \\
& model & shell & $\delta_{i}$ & \multicolumn{3}{c}{ participations $\mu_{i}(\%)$} \\
& $f_{i}(\mathrm{~Hz})$ & $f_{\mathrm{A} i}(\mathrm{~Hz})$ & $(\%)$ & $\chi_{8}$ & $\chi_{9}$ & $\chi_{10}$ & $\chi_{11}$ \\
\hline 1st & 85.793 & 81.477 & 4.07 & 12.6 & 0 & 0 & 0 \\
2nd & 106.64 & 101.77 & 3.80 & 0 & 7.76 & 0 & 0 \\
3rd & 134.54 & 126.34 & 4.91 & 0 & 0 & 97.3 & 0 \\
4th & 194.95 & 190.51 & 2.33 & 0 & 0 & 0 & 100 \\
5th & 202.36 & 202.28 & 0.04 & 0 & 0 & 0 & 100 \\
6th & 216.42 & 225.57 & -4.06 & 0 & 0 & 0 & 100 \\
7th & 243.86 & 240.80 & 1.27 & 0 & 83.5 & 0 & 0 \\
8th & 272.82 & 260.68 & 4.66 & 81.1 & 0 & 0 & 100 \\
9th & 286.84 & 279.35 & 2.68 & 0 & 94.6 & 0 & 0 \\
10th & 310.65 & 307.93 & 0.88 & 0 & 0 & 0 & 100 \\
\hline
\end{tabular}

The results in Table 1 show that the natural frequencies derived from the present model are very close to those from ANSYS shell theory. Moreover, the results provide the fact that distortion modes play important roles even in lower-order vibration modes and big errors may emerge if distortions are ignored.

To further study the dynamic behaviours, Figures 6 and 7 provide the modal shapes concerning the 1st 10th modes. The comparison reconfirms that the present model agrees well with ANSYS shell theory and also proves that the present model can accurately reproduce three-dimensional dynamic behaviours of thin-walled structures.

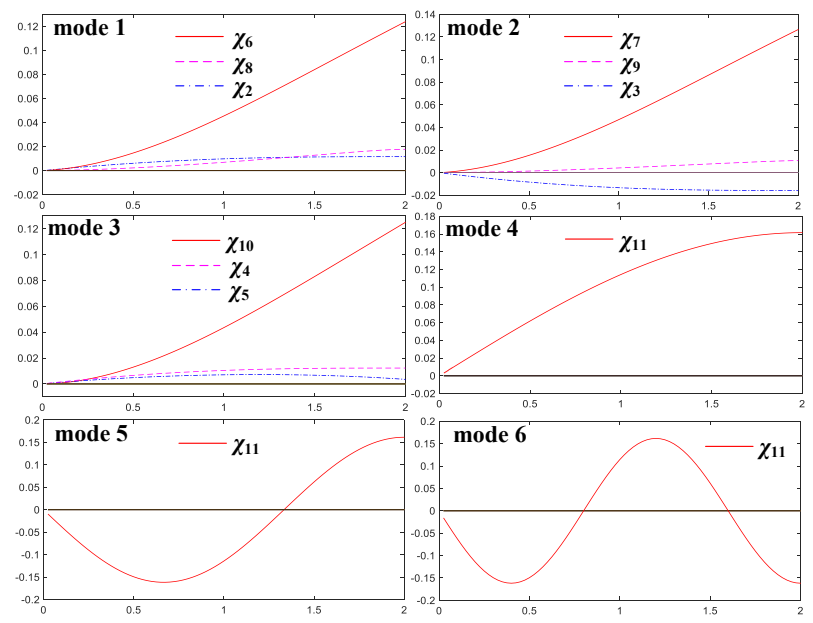

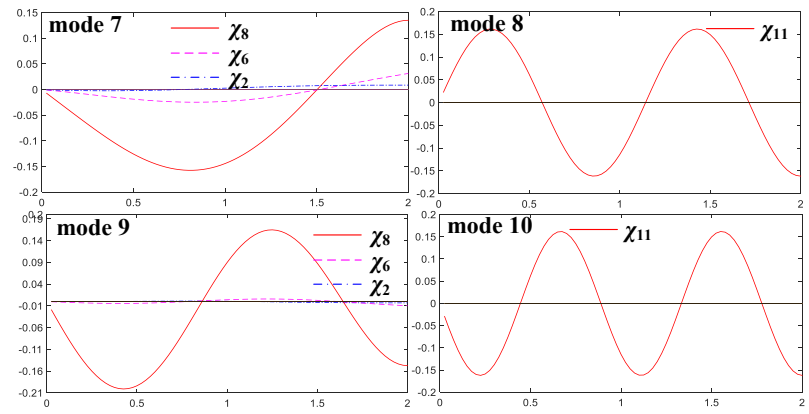

Fig. 6. Longitudinal variations of generalized displacements of the thin-walled structure in the free vibration analyses concerning the first 10 modes.

Besides, it's interesting that distortion $4\left(\chi_{11}\right)$ is not to appear along with classic modes, quite different from other distortions. In this aspect, distortion 4 is more similar to classic modes which plays important role in conventional beam theory. This phenomenon is worth of much attention in the follow-up study.
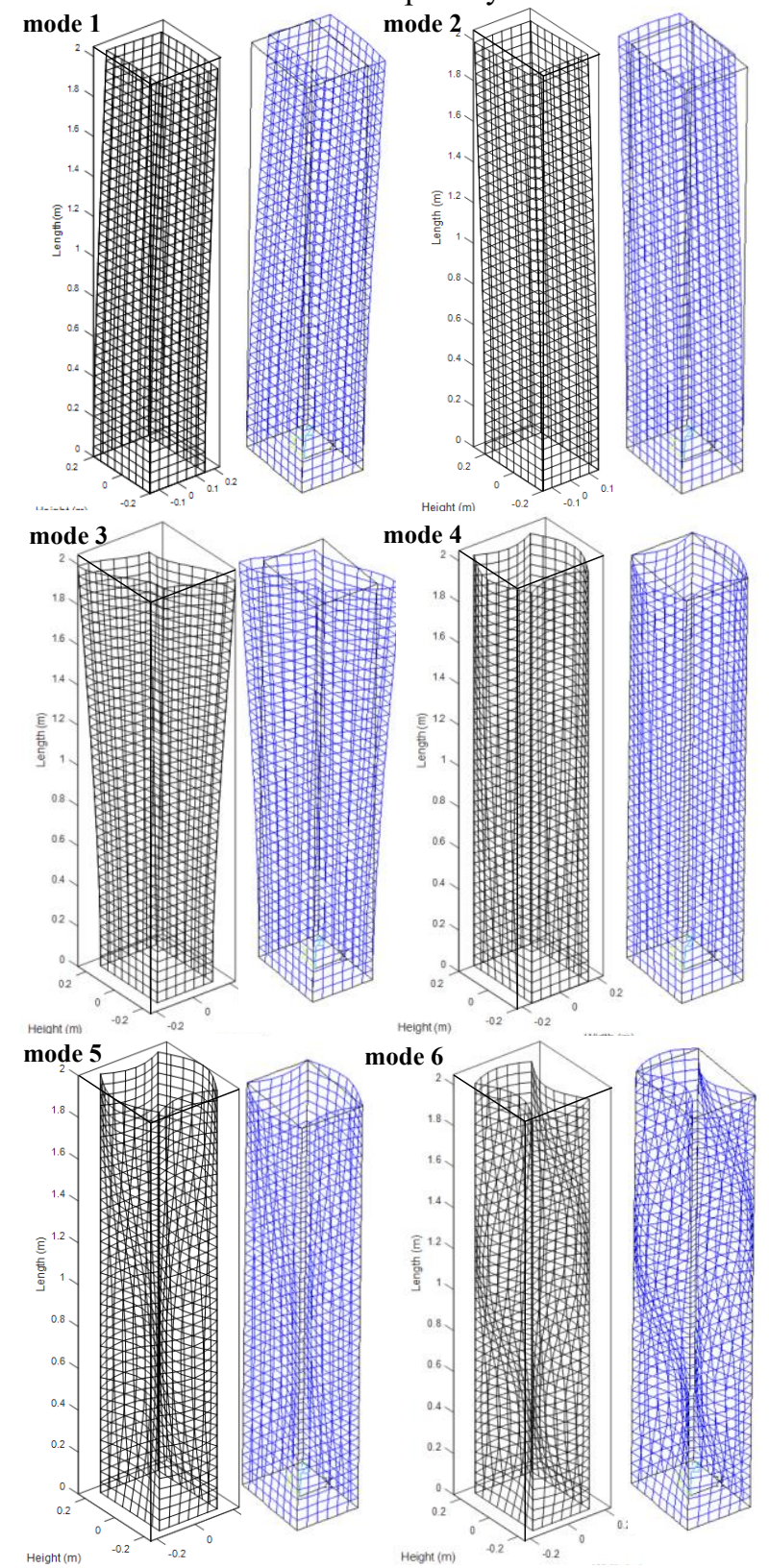


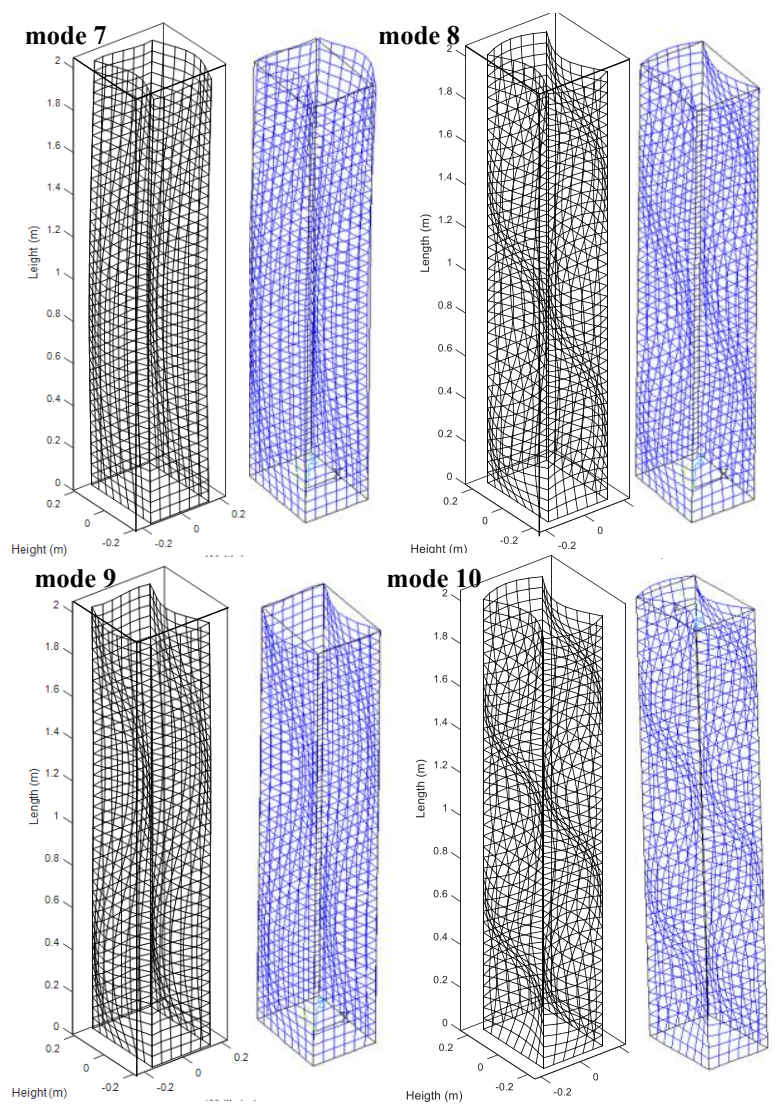

Fig. 7. Comparison of free vibration mode shapes of the thinwalled beam between ANSYS shell model (the right) and present model (the left) concerning the first 10 modes.

\section{Conclusions}

An approach to identify distortion of thin-walled structures has been presented with a one-dimensional beam model established for dynamic analyses. In the process, the sectional in-plane deformation components are first attracted from sectional vibration shapes derived in modal analyses of thin-walled structures based on shell/plate theory. Then the classic mode is deleted from the vibration shapes referring to the minimum value of 2norm with residual deformation components left. And the method of curve fitting is involved to approximate the shape functions of the residual deformation components, which is defined as distortion. The newly identified distortions are employed in one-dimensional model to reproduce three-dimensional behaviours of thin-walled structures. It should be noted that the number of shell-like vibration modes to be studied is adjustable according to the number of distortions in demand. Besides, the approach is not only usable to distortion, but also applicable to warping with further improvement.

To verify the validity, numerical examples are performed on a rectangular cross-section thin-walled structure. Table 1 shows that distortion modes play non negligible roles even in lower-order vibration modes and the natural frequencies derived from the present model are very close to those from ANSYS shell theory with relative differences less than $5 \%$ for the first 10 modes. Figures 6 and 7 reconfirms that the present model agrees well with ANSYS shell theory and also proves that it can reproduce three-dimensional dynamic behaviours of thinwalled structures. The authors plan to refine the procedure for cross-sections with curved walls next.

\section{Acknowledgements}

The authors gratefully acknowledge the financial support of National Natural Science Foundation of China (Grant No. 51805144), Natural Science Foundation of Jiangsu Province (Grant No. BK20170300), the Fundamental Research Funds for the Central Universities, and the Foundation of Changzhou Key Laboratory of Aerial Work Equipment and Intelligent Technology.

\section{References}

1. M. Petrolo, M.H. Nagaraj, I. Kaleel, E. Carrera, Comput. Struct., 206, 54, (2018)

2. R. Gonçalves, M. Ritto-Corrêa, D. Camotim, Comput. Mech., 46, 759, (2010)

3. W. Yu, D.H. Hodges, J. Am. Helicopter Soc., 50, 46, (2005)

4. E. Carrera, M. Petrolo, J. Appl. Mech. - T. ASME, 78, 021013, (2011)

5. Y. Kim, Y.Y. Kim, Int. J. Solids Struct., 40, 6111, (2003)

6. R. Gonçalves, N. Peres, R. Bebiano, D. Camotim, Int. J. Struct. Stab. Dy, 15, 1540022, (2015)

7. S.S. Chen, Z.L. Tian, S.R. Gui, J. Highway Transp. Res. Dev. (English Ed.), 10, 33, (2016)

8. A. Pagani, M. Boscolo, J.R. Banerjee, E. Carrera, J. Sound Vib., 332, 6104, (2013)

9. R. Gonçalves, R. Bebiano, D. Camotim, Thin Wall. Struct., 84, 325, (2014)

10. S. de Miranda, A. Madeo, D. Melchionda, L. Patruno, A.W. Ruggerini, Int. J. Solids Struct., 121, 212, (2017)

11. M. Mohammad-Abadi, A.R. Daneshmehr, Int. J. Eng. Sci., 87, 83, (2015).

12. G. Garcea, R. Gonçalves, A. Bilotta, D. Manta, R. Bebiano, L. Leonetti, D. Magisano, D. Camotim, Thin Wall. Struct., 100, 192, (2016)

13. R.F. Vieira, F.B. Virtuoso, E.B.R. Pereira, Int. J. Solids Struct., 51, 575, (2014).

14. R.F. Vieira, F.B.E Virtuoso, E.B.R. Pereira, Comput. Struct., 147, 68, (2015)

15. R. Bebiano, C. Basaglia, D. Camotim, R. Gonçalves, Thin Wall. Struct., 123, 11, (2018) 